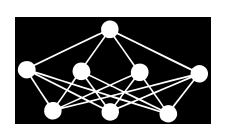

\title{
APPLICATION OF NEURAL NETWORK MODEL FOR PARAMETERS IDENTIFICATION OF NON-LINEAR DYNAMIC SYSTEM
}

\author{
D. Balara, J. Timko, J. Žilková
}

\begin{abstract}
A method for identification of parameters of a non-linear dynamic system, such as an induction motor with saturation effect taken into account, is presented in this paper. Adaptive identifier with structure similar to model of the system performs identification. This identifier can be regarded as a special neural network, therefore its adaptation is based on the gradient descent method and Back-Propagation well known in the neural networks theory. Parameters of electromagnetic subsystems were derived from the values of synaptic weights of the estimator after its adaptation. Testing was performed with simulations taking into account noise in measured quantities. Deviations of identified parameters in case of electrical parameters of the system were up to $1 \%$ of real values. Parameters of non-linear magnetizing curve were identified with deviations up to $6 \%$ of real values. Identifier was able to follow sudden changes of rotor resistance, load torque and moment of inertia.
\end{abstract}

Key words: Neural networks, non-linear dynamic systems, induction machines, identification

Received: October 19, 2012

Revised and accepted: February 25, 2013

\section{Introduction}

Identification has been studied for decades from a variety of viewpoints and research communities, such as statistical regression-estimation, signal processing-filtering, and control engineering-adaptive control. When a priori information about the rules that govern a system either do not exist or are too complex, identification techniques are used to build a model by only observing input-output data. Then, the system is called a 'black box' since the internal behavior is unknown. On the

*D. Balara, J. Timko, J. Žilková

Department of Electrical Engineering and Mechatronics, Technical University of Košice, Letná 9, 04200 Košice, Slovak Republic, E-mail: balarad@vadium.sk, jaroslav.timko@tuke.sk, jaroslava.zilkova@tuke.sk 
contrary, in technical practice we often meet with the case that the system does not behave just like a 'black box', but we have some a priori information about it. In many physical systems our knowledge of mechanical or electrical laws allow us to formulate a model which is the fundamental tool for studying and analysis its properties. However, no matter how deep our physical insight, the parameters of any model present inaccuracies.

In case of induction motor identification, it is possible to assume that some parameters are known, the magnetizing curve is linear, identified motor is at standstill or rotary speed of motor is constant during some time period. Above assumptions simplify identification, but at the same time they limit the number of identifiable parameters or they can be used only in special working modes of the motor. Our aim was to develop an identification procedure of all induction motor parameters, if possible, with non-linear friction [1-3] and saturation effect taken into account [4-8]. An inspiration for handling such a complex system may be found in the artificial neural networks applications, where a complex system is divided into small units - neurons operating according to local-defined rules. If rules are properly designed, network produces required global effect, although operation of each neuron is strictly local. Though the whole network may be strongly non-linear, operating rules of each neuron may be very simple. Proposed identifier of induction motor is based on the same principle. The identifier is constructed as a model of the motor with all above non-linearities incorporated. The identifier is properly divided into neurons. Parameters are replaced with adaptive weights and whole identifier works as a network of several neurons, each of them operating according to local-defined rules. Using stator voltages and rotary speed as inputs and stator currents as demanded outputs, identifier adapts its weights with use of the well-known gradient descent method [9]. The deviations of demanded and actual outputs of identifier are transferred to inner neurons with use of the Back-Propagation algorithm [3]. Many non-linearities and feedbacks influence only input signals of neurons, therefore neurons themselves are linear and their adaptive rules are very simple. Proposed identifier identifies stator and rotor resistances, leakage inductances and course of non-linear magnetizing curve. Testing of the identifier was performed with simulations. To make testing conditions somewhat more similar to reality, Gaussian noise was added to signals supposed to be measured from motor. Structure of the identifier is similar to model of motor in $\alpha \beta$ frame of reference [11]. As a consequence of this transformation, quantities of motor do oscillate and contain more information for the purpose of identification.

\section{Model of Induction Motor}

The two phase electrical equations of an induction motor in an arbitrary frame rotating at a speed $\omega_{a}$ are given by [6]:

$$
\begin{gathered}
\bar{U}_{S}=R_{S} \bar{I}_{S}+\frac{d \bar{\psi}_{S}}{d t}+j \cdot p_{p} \omega_{a} \bar{\psi}_{S} \\
\bar{U}_{R}=\overline{0}=R_{R} \bar{I}_{R}+\frac{d \bar{\psi}_{R}}{d t}+j \cdot p_{p}\left(\omega_{a}-\omega\right) \cdot \bar{\psi}_{R}
\end{gathered}
$$


Balara D. et al.: Application of neural network model for parameters...

where

$$
\begin{gathered}
\bar{\psi}_{S}=L_{\sigma S} \bar{I}_{S}+\bar{\psi}_{m} \text { and } \bar{\psi}_{R}=L_{\sigma R} \bar{I}_{R}+\bar{\psi}_{m} \\
\bar{\psi}_{m}=\bar{F}\left(\bar{I}_{m}\right) \text { and } \bar{I}_{m}=\bar{I}_{S}+\bar{I}_{R} \\
m=\frac{3}{2} p_{p} \operatorname{Im}\left[\bar{\psi}_{S}^{*} \cdot \bar{I}_{S}\right] .
\end{gathered}
$$

Symbol * denotes conjugate vector.

\begin{tabular}{|l|l|}
\hline$I_{S}, I_{R}, I_{m}$ & stator, rotor and magnetizing currents \\
\hline$U_{S}, U_{R}$ & stator and rotor voltages \\
\hline$\psi_{S}, \psi_{R}, \psi_{m}$ & stator, rotor and mutual fluxes \\
\hline$p_{p}$ & number of the pole pairs \\
\hline$\omega_{a}$ & angular speed \\
\hline$\omega$ & mechanical angular speed \\
\hline$m$ & torque \\
\hline$L_{\sigma S}, L_{\sigma R}$ & leakage inductances of stator and rotor \\
\hline$R_{S}, R_{R}$ & stator and rotor resistance \\
\hline
\end{tabular}

Tab. I Used symbols.

In stator coordinate system [11] $\alpha \beta$ frame of reference $\omega_{a}=0$. Based on (1), we obtain:

$$
\begin{gathered}
\bar{U}_{S}=R_{S} \bar{I}_{S}+\frac{d \bar{\psi}_{S}}{d t} \\
\bar{U}_{R}=\overline{0}=R_{R} \bar{I}_{R}+\frac{d \bar{\psi}_{R}}{d t}-j \cdot p_{p} \cdot \omega \cdot \bar{\psi}_{R}
\end{gathered}
$$

and the transformation of three-phases stator voltages into stationary $\alpha, \beta$ reference frame is by [11]:

$$
u_{\alpha 1}=\frac{1}{3}\left(2 u_{a}-u_{b}-u_{c}\right) \text { and } u_{\beta 1}=\frac{1}{\sqrt{3}}\left(u_{b}-u_{c}\right) .
$$

From (6) and (7) follows that

$$
\frac{d \bar{\psi}_{S}}{d t}=\bar{U}_{S}-R_{S} \bar{I}_{S} \text { and } \frac{d \bar{\psi}_{R}}{d t}=-R_{R} \bar{I}_{R}+j \cdot p_{p} \cdot \omega \cdot \bar{\psi}_{R}
$$

and in component form

$$
\begin{gathered}
{\left[\begin{array}{c}
\frac{d \psi_{\alpha 1}}{d t} \\
\frac{d \psi_{\beta 1}}{d t}
\end{array}\right]=\left[\begin{array}{l}
u_{\alpha 1} \\
u_{\beta 1}
\end{array}\right]-R_{S}\left[\begin{array}{l}
i_{\alpha 1} \\
i_{\beta 1}
\end{array}\right]} \\
{\left[\begin{array}{c}
\frac{d \psi_{\alpha 2}}{d t} \\
\frac{d \psi_{\beta 2}}{d t}
\end{array}\right]=-R_{R}\left[\begin{array}{l}
i_{\alpha 2} \\
i_{\beta 2}
\end{array}\right]+p_{p} \cdot \omega\left[\begin{array}{l}
-\psi_{\beta 2} \\
\psi_{\alpha 2}
\end{array}\right]}
\end{gathered}
$$

From equation (3): 


$$
\bar{I}_{S}=\frac{\bar{\psi}_{S}-\bar{\psi}_{m}}{L_{\sigma S}} \text { and } \bar{I}_{R}=\frac{\bar{\psi}_{R}-\bar{\psi}_{m}}{L_{\sigma R}}
$$

According to [4], where authors considered controlled induction motor, the vector version of the saturation function in (3) is defined as $\bar{F}: \Re^{2} \rightarrow \Re^{2}$ such that $\left\|\bar{\psi}_{m}\right\|=f\left(\left\|\bar{I}_{m}\right\|\right)$ and $\psi_{\alpha m} / \psi_{\beta m}=i_{\alpha m} / i_{\beta m}$. Function $f: \Re \rightarrow \Re$ is a scalar saturation function that only affects the magnitude of the mutual flux $\bar{\psi}_{m}$ so that $\bar{\psi}_{m}$ a $\bar{I}_{m}$ have the same angle. According to [10] the time constant $T_{m g}$, of leeway of flux after magnetizing current in dynamo sheets, is not zero, but approximately $0.016 \mathrm{~ms}$.

Let us denote

$$
I_{m}=\left\|\bar{I}_{m}\right\|=\sqrt{I_{\alpha m}^{2}+I_{\beta m}^{2}} .
$$

Components of mutual flux may be written by [4] as follows:

$$
\psi_{\alpha m}=i_{\alpha m} \frac{f\left(I_{m}\right)}{I_{m}} \quad \psi_{\beta m}=i_{\beta m} \frac{f\left(I_{m}\right)}{I_{m}} .
$$

According to [10] the first order inertias with very small time constant $T_{m g}$ were included into the model. Including by [10] the first-order inertias into (12):

$$
\begin{aligned}
& \frac{d \psi_{\alpha m}}{d t}=\frac{1}{T_{m g}} i_{\alpha m} \frac{f\left(I_{m}\right)}{I_{m}}-\frac{1}{T_{m g}} \psi_{\alpha m} \\
& \frac{d \psi_{\beta m}}{d t}=\frac{1}{T_{m g}} i_{\beta m} \frac{f\left(I_{m}\right)}{I_{m}}-\frac{1}{T_{m g}} \psi_{\beta m} .
\end{aligned}
$$

The mechanical equation can be expressed as:

$$
m-m_{L} \cdot \operatorname{sgn}(\omega)-m_{f}=J \frac{d \omega}{d t},
$$

where $m, m_{L}$ and $m_{f}$ are motor, load and friction torque and $J$ is moment of inertia.

Substituting (2) into (5), we obtain:

$$
m=\frac{3}{2} p_{p}\left[i_{\beta 1} \cdot \psi_{\alpha m}-i_{\alpha 1} \cdot \psi_{\beta m}\right] .
$$

Fig. 1 shows transformation of three-phases stator voltages into stationary $\alpha, \beta$ reference frame by (8).

The discrete model of an induction motor with non-linear magnetic circuit is represented in Figs. 2-4, where symbol $\square$ denotes numerical integrator. Fig. 2 and 3 shows discrete model of stator and rotor of an induction motor by (3), (4) and (9).

Fig. 4 shows discrete model of magnetizing subsystem of an induction motor by (10)-(13). Function $g$ comprises non-linear magnetizing curve, the square root function and dividing by module of $I_{m}$. 
Balara D. et al.: Application of neural network model for parameters...

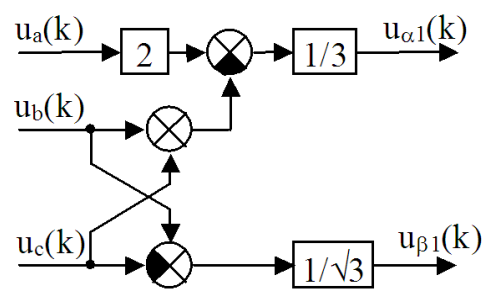

Fig. 1 Transformation of phase voltages into $\alpha, \beta$ frame of reference.

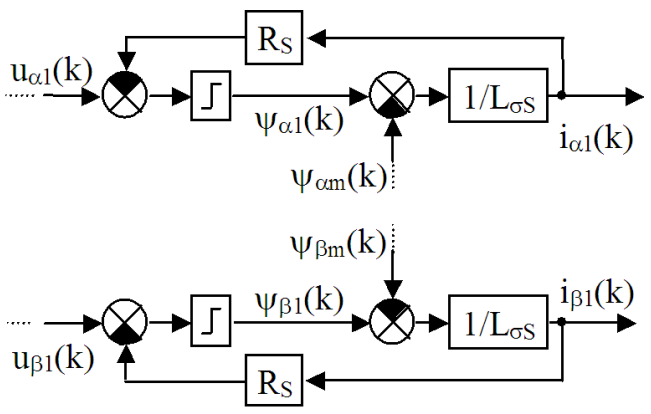

Fig. 2 Model of stator of an induction motor.

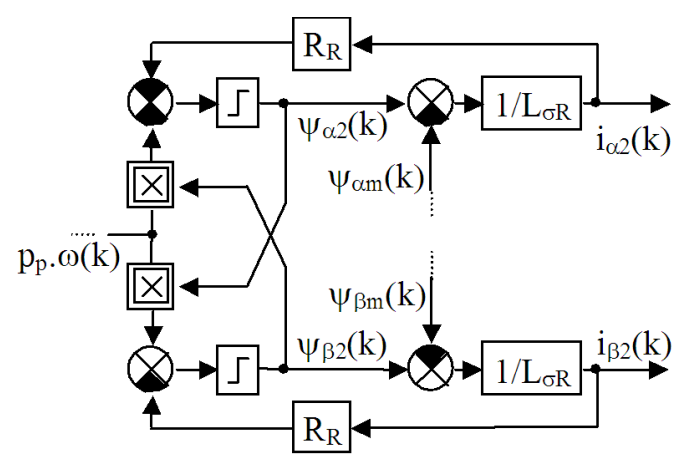

Fig. 3 Model of rotor of an induction motor.

\section{Neural Identifier}

General structure of the proposed identifier is shown in Fig. 5. Figs. 6-8 depict detailed structure of the identifier. After adaptation, identified parameters can be derived from particular weights of the identifier based on its similarity to identified motor's structure.

Symbol * denotes identified signals.

In case of ideal adaptation weights for neurons $\mathrm{N} 1$ and N2 by Fig. 6 is $w_{1}^{N 1}=$ $w_{1}^{N 2}=-R_{S}$, and $w_{O}^{N 1}=w_{O}^{N 2}=1 / L_{\sigma S}$. The output $o$ of the neuron N1 is determined by (17) and output of neuron N2 analogically. 


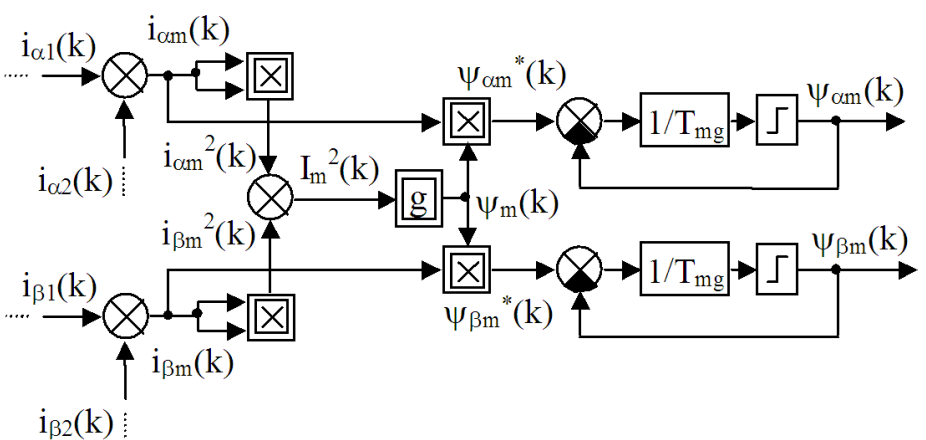

Fig. 4 Model of magnetizing subsystem.

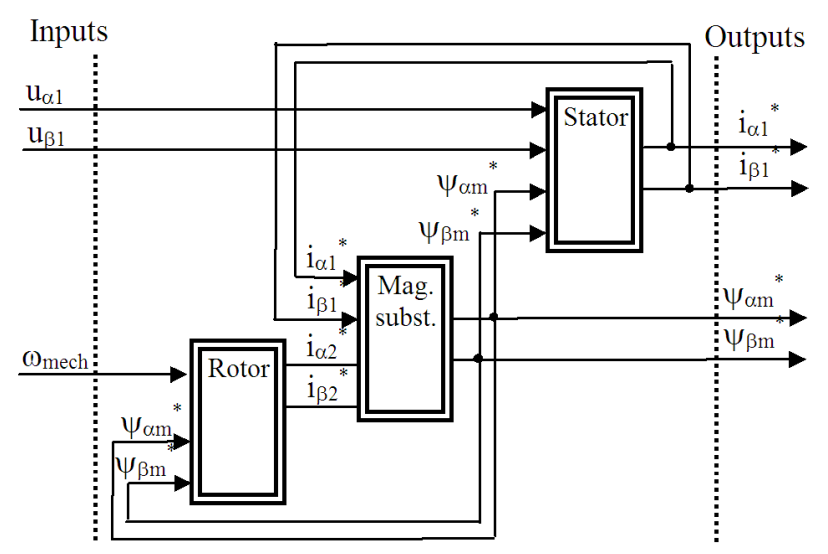

Fig. 5 General scheme of electromagnetic identifier.

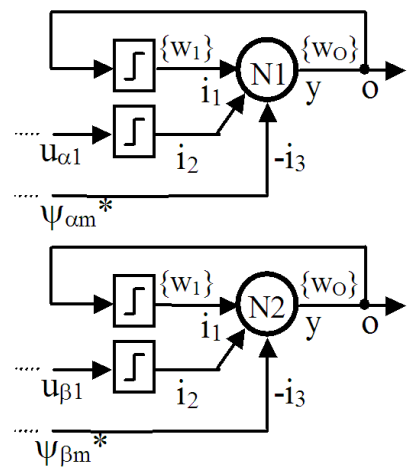

Fig. 6 Neural identifier of stator.

In case of ideal adaptation weights for neurons N3, N4, N5 and N6 is $w_{O}^{N 4}=$ $w_{O}^{N 6}=1 / L_{\sigma R}$ and $w_{1}^{N 3}=w_{1}^{N 5}=-R_{R}$. The outputs of the neurons are determined analogically with the neuron N1. 
Balara D. et al.: Application of neural network model for parameters...

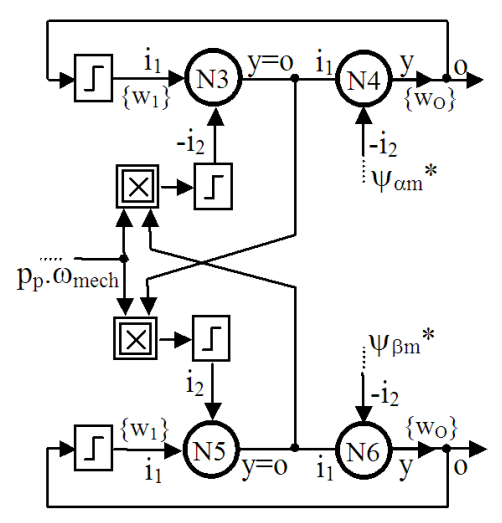

Fig. 7 Neural identifier of rotor.

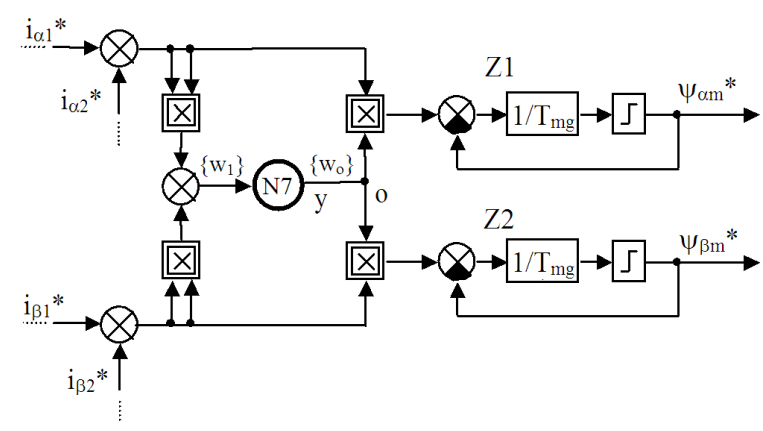

Fig. 8 Neural identifier of magnetizing subsystem.

Neural identifier of magnetizing subsystem is shown in Fig. 8. The neuron N7 represents the magnetizing curve. In case of ideal adaptation of weights for the neuron $\mathrm{N} 7, w_{1}^{N 7}=-d$ and $w_{O}^{N 7}=c$. Z1 and Z2 are first order inertias by (13).

\section{Rules of adaptation}

Identifiers of stator and rotor contain branches with identical parameters. During adaptation, however, signals in particular branches are different and their information value from identification point of view is different too. Therefore, adaptation of weights in one of the branches is usually slower and it may even stop at a certain level. It is useful to perform adaptation so that values of corresponding weights are bound somehow in order to preserve their similar values. In this case, this aim was achieved by averaging corresponding weights after each step of adaptation. This approach ensures identical values of corresponding weights in each step and, at the same time, the information value of signals in both branches is used for adaptation.

To derive adaptation rules for all neurons, the gradient descent method and Back-Propagation algorithm were used. Let there be objective function $E^{=\frac{1}{2}} e^{2}$, where $e-$ is the deviation between required and actual output of the neuron. 


\section{For deviation and error function of neuron N1}

$$
\begin{gathered}
e^{N 1}=i_{\alpha 1}-o^{N 1} \quad E^{N 1}=\frac{1}{2}\left(e^{N 1}\right)^{2} \\
o^{N 1}=w_{O}^{N 1}\left(w_{1}^{N 1} \cdot i_{1}^{N 1}+1 \cdot i_{2}^{N 1}-1 . i_{3}^{N 1}\right)=w_{O}^{N 1} \cdot y^{N 1} .
\end{gathered}
$$

According to the gradient descent algorithm for weights adaptation of neuron N1

$$
\begin{gathered}
\frac{d w_{O}^{N 1}}{d t}=-\eta_{O}^{N 1} \frac{\partial E^{N 1}}{\partial w_{O}^{N 1}}=\eta_{O}^{N 1} \cdot e^{N 1} \cdot y^{N 1} \\
\frac{d w_{1}^{N 1}}{d t}=-\eta_{1}^{N 1} \frac{\partial E^{N 1}}{\partial w_{1}^{N 1}}=\eta_{1}^{N 1} \cdot e^{N 1} \cdot w_{O}^{N 1} \cdot i_{1}^{N 1},
\end{gathered}
$$

where $i_{n}$ is $n$-th input of the neuron and $\eta$ is the rate of the adaptation.

Analogically for neuron N2

$$
\begin{gathered}
e^{N 2}=i_{\beta 1}-o^{N 2} \\
\frac{d w_{O}^{N 2}}{d t}=\eta_{O}^{N 2} e^{N 2} y^{N 2} \text { and } \frac{d w_{1}^{N 2}}{d t}=\eta_{1}^{N 2} e^{N 2} w_{O}^{N 2} i_{1}^{N 2} .
\end{gathered}
$$

\section{For neurons N4 and N6}

$$
o^{N 4}=w_{O}^{N 4}\left(1 \cdot i_{1}^{N 4}-1 \cdot i_{2}^{N 4}\right)=w_{O}^{N 4} \cdot y^{N 4} .
$$

If $e^{N 4}$ would be known then

$$
\begin{gathered}
E^{N 4}=\frac{1}{2}\left(e^{N 4}\right)^{2} \\
\frac{d w_{O}^{N 4}}{d t}=-\eta_{O}^{N 4} \frac{\partial E^{N 4}}{\partial w_{O}^{N 4}}=\eta_{O}^{N 4} \cdot e^{N 4} \cdot y^{N 4} .
\end{gathered}
$$

In order to derive adaptation rule we can consider the Z1 inertia with a very small time constant to have almost the same effect as non-inertia element with gain 1. Neuron N4 affects the operation of neuron N1, therefore:

$$
\begin{gathered}
o^{N 1} \cong w_{O}^{N 1}\left(w_{1}^{N 1} \cdot i_{1}^{N 1}+i_{2}^{N 1}-o^{N 7}\left(o^{N 4}+o^{N 1}\right)\right) \\
e^{N 1}=r^{N 1}-o^{N 1} \\
\frac{d w_{O}^{N 4}}{d t}=-\eta_{O}^{N 4} \frac{\partial E^{N 1}}{\partial w_{O}^{N 4}}=\eta_{O}^{N 4} e^{N 1} w_{O}^{N 1}\left(-o^{N 7} y^{N 4}\right) .
\end{gathered}
$$

From (23) and (26) follows that

$$
e^{N 4}=-e^{N 1} w_{O}^{N 1} o^{N 7} .
$$

Analogically

$$
e^{N 6}=-e^{N 2} w_{O}^{N 2} o^{N 7} \text { and } \frac{d w_{O}^{N 6}}{d t}=\eta_{O}^{N 6} e^{N 6} y^{N 6} .
$$


Balara D. et al.: Application of neural network model for parameters...

\section{For neurons N3 a N5}

$$
o^{N 3}=w_{1}^{N 3} \cdot i_{1}^{N 3}-1 \cdot i_{2}^{N 3} .
$$

If $e^{N 3}$ would be known then

$$
\begin{gathered}
E^{N 3}=\frac{1}{2}\left(e^{N 3}\right)^{2} \\
\frac{d w_{1}^{N 3}}{d t}=-\eta_{1}^{N 3} \frac{\partial E^{N 3}}{\partial w_{1}^{N 3}}=\eta_{O}^{N 3} \cdot e^{N 3} \cdot i_{1}^{N 3} .
\end{gathered}
$$

Since neuron N3 affects the neuron N4, it can be written:

$$
\begin{gathered}
o^{N 4}=w_{O}^{N 4}\left(1 \cdot o^{N 3}-1 \cdot i_{2}^{N 4}\right) \\
e^{N 4}=r^{N 4}-o^{N 4} \\
\frac{d w_{1}^{N 3}}{d t}=-\eta_{1}^{N 3} \frac{\partial E^{N 4}}{\partial w_{1}^{N 3}}=-\eta_{1}^{N 3} \cdot \frac{\partial E^{N 4}}{\partial e^{N 4}} \cdot \frac{\partial e^{N 4}}{\partial w_{1}^{N 3}}=\eta_{1}^{N 3} \cdot e^{N 4} \cdot w_{O}^{N 4} \cdot i_{1}^{N 3} .
\end{gathered}
$$

From (31) and (33) follows that

$$
e^{N 3}=e^{N 4} \cdot w_{O}^{N 4} .
$$

Analogically

$$
e^{N 5}=e^{N 6} w_{O}^{N 6} \text { and } \frac{d w_{1}^{N 5}}{d t}=\eta_{O}^{N 5} e^{N 5} i_{1}^{N 5} .
$$

\section{For neuron N7}

$$
o^{N 7}=w_{O}^{N 7} \cdot \frac{1-\exp \left(w_{1}^{N 7} \cdot i_{1}^{N 7}\right)}{i_{1}^{N 7}}=w_{O}^{N 7} \cdot y^{N 7} .
$$

If $e^{N 7}$ would be known then

$$
\begin{gathered}
E^{N 7}=\frac{1}{2}\left(e^{N 7}\right)^{2} \\
\frac{d w_{O}^{N 7}}{d t}=-\eta_{O}^{N 7} \frac{\partial E^{N 7}}{\partial w_{O}^{N 7}}=\eta_{O}^{N 7} \cdot e^{N 7} \cdot y^{N 7} \\
\frac{d w_{1}^{N 7}}{d t}=-\eta_{O}^{N 7} \frac{\partial E^{N 7}}{\partial w_{1}^{N 7}}=-\eta_{O}^{N 7} e^{N 7} w_{O}^{N 7} \exp \left(w_{1}^{N 7} i_{1}^{N 7}\right) .
\end{gathered}
$$

Neuron N4 influences neurons N1 a N2. If for derivation of adaptive rules we substitute inertias Z1 a Z2 with simple gains 1 then following equations can be written:

$$
\begin{aligned}
o^{N 1} & =w_{O}^{N 1}\left(w_{1}^{N 1} \cdot i_{1}^{N 1}+i_{2}^{N 1}-o^{N 7} \cdot\left(o^{N 1}+o^{N 4}\right)\right) \\
o^{N 2} & =w_{O}^{N 2}\left(w_{1}^{N 2} \cdot i_{1}^{N 2}+i_{2}^{N 2}-o^{N 7} \cdot\left(o^{N 2}+o^{N 6}\right)\right) \\
& e^{N 1}=r^{N 1}-o^{N 1} \text { and } e^{N 2}=r^{N 2}-o^{N 2}
\end{aligned}
$$




$$
\begin{aligned}
\frac{d w_{O}^{N 7}}{d t} & =-\eta_{O}^{N 7} \frac{\partial E^{N 1}}{\partial w_{O}^{N 7}}=-\eta_{O}^{N 7} \frac{\partial E^{N 1}}{\partial e^{N 1}} \frac{\partial e^{N 1}}{\partial o^{N 7}} \frac{\partial o^{N 7}}{\partial w_{O}^{N 7}}= \\
& =\eta_{O}^{N 7} \cdot e^{N 1} \cdot w_{O}^{N 1}\left(-\left(o^{N 1}+o^{N 4}\right)\right) \cdot y^{N 7} \\
\frac{d w_{O}^{N 7}}{d t} & =-\eta_{O}^{N 7} \frac{\partial E^{N 2}}{\partial w_{O}^{N 7}}=-\eta_{O}^{N 7} \frac{\partial E^{N 2}}{\partial e^{N 2}} \frac{\partial e^{N 2}}{\partial o^{N 7}} \frac{\partial o^{N 7}}{\partial w_{O}^{N 7}} \\
& =\eta_{O}^{N 7} \cdot e^{N 2} \cdot w_{O}^{N 2}\left(-\left(o^{N 2}+o^{N 6}\right)\right) \cdot y^{N 7}
\end{aligned}
$$

From a comparison of (38) and (43), (38) and (44) follows that error signal of neuron $\mathrm{N} 7$ can be defined in two ways:

$$
\begin{aligned}
& e^{N 7}=-e^{N 1} \cdot w_{O}^{N 1} \cdot\left(o^{N 1}+o^{N 4}\right) \\
& e^{N 7}=-e^{N 2} \cdot w_{O}^{N 2} \cdot\left(o^{N 2}+o^{N 6}\right) .
\end{aligned}
$$

\section{Identification of electromagnetic parameters}

According to [10] the time constant of leeway of flux after magnetizing current is approximately $0.016 \mathrm{~ms}$, therefore the time constant of magnetizing circuit was considered to be $T_{m g} *=0.016 \mathrm{~ms}$. Other parameters of a squirrel-cage induction motor used in the simulation were: $m_{L}=0 \mathrm{Nm}, b=0 \mathrm{Nms}, U_{z}=190 \mathrm{~V}, f=50 \mathrm{~Hz}$, $R_{S}=0.181 \Omega, R_{R}=0.161 \Omega, J=0.11 \mathrm{kgm}^{2}, p_{p}=2, L_{\sigma S}=0.00183 \mathrm{H}, L_{\sigma R}=0.00183 \mathrm{H}$, $\left\|\bar{\psi}_{m}\right\|=c\left(1-e^{-d\left\|\bar{I}_{m}\right\|}\right), c=0.32 \mathrm{~Wb}, d=0.2$.

Considered was motor loaded with friction with various components [11] and passive load torque. Measured signals of motor, i.e. stator voltages and currents and rotary speed, were simulated with Gaussian noise added. Parameters of induction motor may vary in time and they can considerably affect the quality of control. Therefore, it is desirable in many cases to use the on-line identification of the rotor resistance [3], [12-15] which varies with temperature of motor. Though its change is continuous, the sudden change of resistance value was considered in testing in order to investigate behavior of the identifier. As the proposed identifier does not identify the time constant of magnetizing circuit, it must be assumed that exact value of this time constant is not known. Therefore, robustness of the identifier is tested with respect to inaccurate value of time-constant in the identifier.

\section{Conditions of simulations}

- Loaded motor ( $b=0.1 \mathrm{Nms}, M_{L}=5 \mathrm{Nm}$ )

- Noisy input signals of identifier

- Non-linear magnetizing curve of motor and identifier

- Inaccurate magnetizing time constant of identifier $T_{m g} *=0.000024$ s, i.e. 150 $\%$ of accurate value used in model of motor

- Sudden change of rotor resistance to $0.19 \Omega$, i.e. $118 \%$ of original value after 2000 periods 
Balara D. et al.: Application of neural network model for parameters...

- 5 filters added to identifier to filter noisy inputs

- Noise: stator currents noise max. $\pm 5 \mathrm{~A}$, stator voltages noise max. $\pm 2 \mathrm{~V}$, speed noise max. \pm 2 rad.s ${ }^{-1}$

- Steps for adaptation of weights were constant and they were tuned experimentally

- Initial values of weights were chose to be of same scale and sign as expected identified values

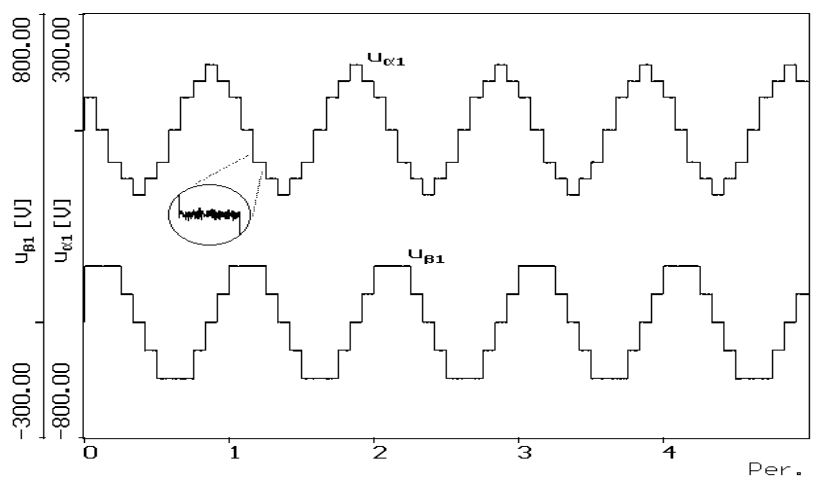

Fig. 9 Stator voltages of the motor.

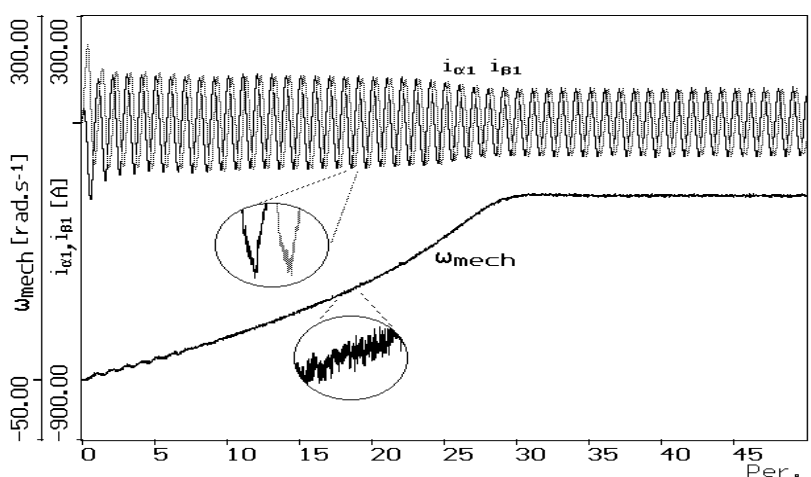

Fig. 10 The training set of the identifier contains 50 periods of stator voltages, currents and rotary speed of the motor.

Tab. II contains values of identified parameters after 8000 periods, i.e. 160 repetition of the training set.

In spite of noise, but with respect to the fact that simulations performed testing, identified parameters are rather accurate. A larger deviation $-4.9950 \%$ occurred when identifying steepness of magnetizing curve $d$, which may be caused by quick transition of motor into the saturated part of the magnetizing curve and consequent 


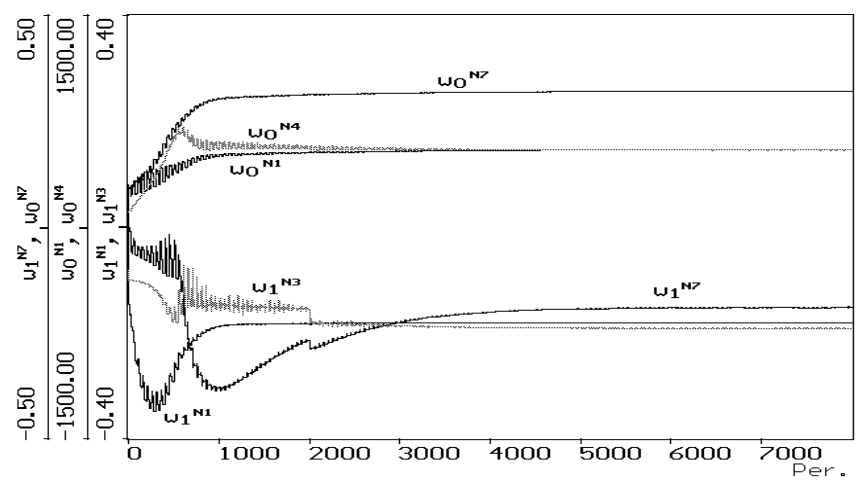

Fig. 11 Adaptation of weights.

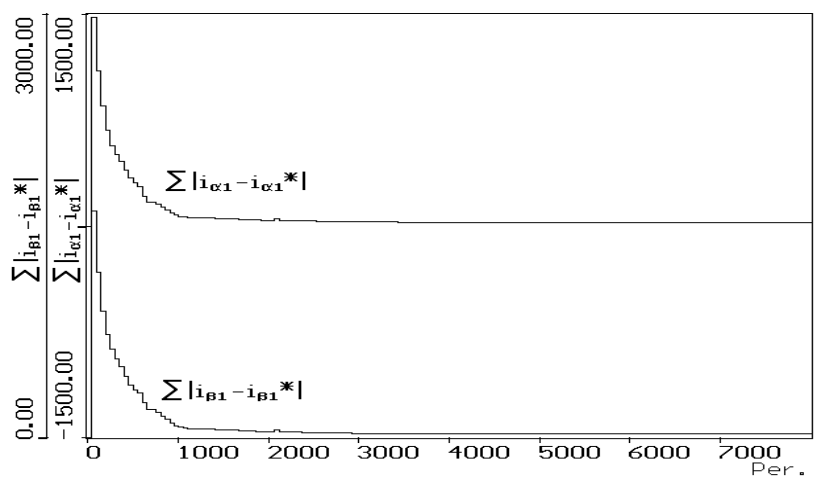

Fig. 12 Average absolute deviations of N1 and N2 outputs during adaptation.

\begin{tabular}{|l|l|l|l|}
\hline Parameter & Identified Value & Real Value & Deviation \\
\hline$R_{S}$ & $0.18088 \Omega$ & $0.181 \Omega$ & $-0.0663 \%$ \\
\hline$R_{R}$ & $0.19138 \Omega$ & $0.190 \Omega$ & $0.7263 \%$ \\
\hline$L_{\sigma S}$ & $0.0018279 \mathrm{H}$ & $0.00183 \mathrm{H}$ & $-0.1156 \%$ \\
\hline$L_{\sigma R}$ & $0.0018279 \mathrm{H}$ & $0.00183 \mathrm{H}$ & $-0.1156 \%$ \\
\hline$d$ & 0.19001 & 0.2 & $-4.9950 \%$ \\
\hline$c$ & 0.32018 & $0.32 \mathrm{~Wb}$ & $0.0180 \%$ \\
\hline
\end{tabular}

Tab. II Identified electromagnetic parameters.

lack of information about non-saturated part of the curve. As can be seen in Fig. 11, reaction of $w_{1}^{N 3}$ weight of the identifier on changed value of rotor resistance was very fast with respect to the dynamic of the whole adaptation process. Noise of measured signals enriches the frequency spectrum of input signals of the identifier, which may help identification but, at the same time, it may endanger its stability.

Similar results were obtained with $T_{m g}^{*}=0.000012 \mathrm{~s}$, i.e. $75 \%$ of value used in motor model. 
Balara D. et al.: Application of neural network model for parameters...

\section{Conclusion}

Deviations of identified parameters in case of electrical parameters of induction motor were up to $1 \%$ of real values. Parameters of non-linear magnetizing curve were identified with deviations up to $6 \%$. The identifier was able to follow sudden changes of rotor resistance, load torque and moment of inertia. All testing was performed with simulations, with a rather strong noise added to quantities, which would be measured from motor in the real application. The proposed identifier contains 7 neurons, most of which are linear. Therefore, adaptation rules are very simple. Identification lasts several thousands of periods of stator voltage, which means several hundreds repetitions of training set. As all repeated training sets were the same, it is possible to generate just a single training set from motor's quantities and repeat it. Adaptation of neurons was done with constant steps whose values were found experimentally. It is probable, therefore, that with optimized steps varying during adaptation the identification process may be much faster.

\section{Acknowledgement}

This work was supported by the Slovak Research and Development Agency under the contract No. APVV-0185-10 and R\&D operational program Center of excellence of power electronics systems and materials for their components No. OPVaV-2008/2.1/01-SORO, ITMS 26220120003 funded by European Regional Development Fund (ERDF).

\section{References}

[1] Beineke S., Schütte F., Grotstollen H.: Comparison of methods for state estimation and online identification in speed and position control loops, Proceedings of the conference, EPE'97, Trondheim, 1997, pp. 3364-3369.

[2] Frenz T., Schröder D.: On line identification and compensation of friction influence of feed drives of machine tools, Proceedings of the conference, EPE'97, Trondheim, 1997, pp. 39273932 .

[3] Timko J., Žilková J., Balara D.: Applications of artificial neural networks in electrical drives. Calypso s.r.o., (in Slovak), Košice, 2002, p. 239.

[4] Hossam A. Abdel Fattah, Kenneth A. Loparo, Hassan M. Emara: Induction motor control system performance under magnetic saturation, American control conference 1999, San Diego, CA, 1999.

[5] Krzeminski Z.: Estimation of induction motor speed in fuzzy logic system, Proceedings of the conference, EPE'97, Trondheim, 1997, pp. 3563-3568.

[6] Levi E.: A unified approach to main flux saturation modelling in d-q axis models of induction machines, IEEE Transactions on Energy Conversion, 10, 10, 1995, pp. 455-461.

[7] Sullivan C. R., Sanders S. R.: Models for induction machines with magnetic saturation on the main flux path, IEEE Transactions on Industrial Applications, 31, 4, 1995, pp. 907-917.

[8] Valouch V.: Iron saturation respect of induction machine for vector based control algorithms, (in Czech), Elektrotechnický obzor 1990, No. 5, 1990.

[9] Baldi P.: Gradient Descent Learning Algorithm Overview: A General dynamical systems perspective, IEEE Transactions on Neural Networks, 6, 1, January 1995, pp. 182-195.

[10] Haňka L.: Theory of electromagnetic field, Praha 1975, SNTL-Alfa, 1975, p. 429.

[11] Timko J., Žilková J., Girovský P.: Electrical drives. TU Košice, CD-ROM, 2007, p. 149. 


\section{Neural Network World 2/13, 103-116}

[12] García-Cerrada A., Zamora J. L.: On-line rotor-resistance estimation for induction motors, Proceedings of the conference, EPE'97, Trondheim, 1997, pp. 1542-1547.

[13] Tungpimolrut K., Fukao T., Peng F. Z.: A robust rotor time constant estimation method for vector control of induction motor under any operating condition, Proceedings of the IECON '94, Bologna 1994, 1994, pp. 275-280.

[14] Žilková J., Timko J.: On-line estimation of quantities using artificial neural networks. Acta Technica Č́SAV 47, No. 3, 2002, pp. 305-315.

[15] Brandštetter P., Škuta O.: Rotor time constant adaptation using radial basis function network. In 13th International Power electronics and motion control conference EPE-PEMC 2008, Poznaň, Poland, 2008, paper No. 131, ISBN 978-1-4244-1742-1/IEEE catalog Number CFP0834A-CDR.

[16] Perduková D., Fedor P.: The Identification of Induction Motor Inner Values. In: Proc. of International Conference on Applied Electrical Engineering and Informatics AEI - 2012, Germany, 2012, pp. 76-80.

[17] Hraško M., Vittek J., Havrila R., Lokseninec I.: Rotor flux observer in pseudo-sliding mode for vector controlled induction motor drives,EPE-PEMC 2006, Portorož, Slovenia, 08-09, 2006, pp. 1133-1136.

[18] Timko J., Žilková J., Girovský P.: Modelling and control of electrical drives by neural networks. C-Press, (in Slovak), Košice, 2009, p. 201. 